\title{
tRNA queuosine modification enzyme modulates the growth and microbiome recruitment to breast tumors
}

\section{Jilei Zhang}

University of Illinois at Chicago College of Medicine

\section{Rong Lu}

University of Illinois at Chicago College of Medicine

\section{Yong-Guo Zhang}

University of Illinois at Chicago College of Medicine

\section{Żaneta Matuszek}

UChicago Medicine

\section{Yinglin Xia}

University of Illinois at Chicago College of Medicine

\section{Tao Pan}

UChicago Medicine

Jun Sun ( $\nabla$ junsun7@uic.edu )

University of Illinois at Chicago College of Medicine https://orcid.org/0000-0001-7465-3133

\section{Research}

Keywords: Breast cancer, breast microbiome, gut microbiome, inflammation, QTRT1, permeability, proliferation, tight junctions

Posted Date: January 17th, 2020

DOI: https://doi.org/10.21203/rs.2.21087/v1

License: (c) (1) This work is licensed under a Creative Commons Attribution 4.0 International License. Read Full License 


\section{Abstract}

Background: Transfer RNA (tRNA) queuosine (Q)-modifications occur specifically in 4 cellular tRNAs at the wobble anticodon position. tRNA Q-modification in human cells depends on the gut microbiome because the microbiome product queuine is required for its installation by the enzyme queuine tRNA ribosyltransferase catalytic subunit 1 (QTRT1) encoded in the human genome. Although tRNA Qmodification has been studied a long time regarding its properties in decoding and tRNA fragment generation, how QTRT1 affects tumorigenesis is still poorly understood.

Results: We generated single clones of QTRT1-knockout breast cancer MCF7 cells using Double Nickase Plasmid. The impacts of QTRT1-delection on cell proliferation and migration in vitro were evaluated using cell culture, while the regulations on tumor growth in vivo were evaluated using xenograft BALB/c nude mouse model. We found that QTRT1 completely deleted from human breast cancer MCF7 cells could change the functions of regulation genes which are critical in cell proliferation, tight junction formation, and migration in human breast cancer cells in vitro and a breast tumor mouse model in vivo. We also found that microbiome maybe involved in the breast cancer development in vivo.

Conclusions: Our results demonstrate that the QTRT1 gene and microbiome play a critical role in breast cancer development.

\section{Background}

tRNAs read the genetic code and are essential for the proliferation, fitness, and adaptation of the cells in the body. Emerging roles of tRNA in various human diseases have been implicated through genetic mutations, post-transcriptional modifications, overexpression, and RNA-protein interactions. tRNA derived small RNA fragments (tRFs) have been shown to be associated with breast cancer in the extracellular vehicles and in the blood, indicating that tRFs may be useful as biomarkers for breast cancer progression and treatment [1-4]. Both tRNA and tRFs function in cells, and in turn their effect on breast cancer tumor development and progression may be modulated by a microbiome-dependent, queuosine (Q)-tRNA modifications. tRNA Q-modification has been associated with various forms of tumors [5]. For example, the severity of liver tumorigenesis was found to be inversely proportional to the amount of Qmodifications in total cellular tRNAs. On the other hand, other tumor types can show the opposite trends. In particular, the potential role of microbiome/Q through tRNA modification has not been explored in breast cancer, although the lack of salvage capabilities following turnover of Q-modified tRNA was said to account for the effect of 50\%-60\% Q-deficiency in the MCF7 breast adenocarcinoma cells [6].

Queuosine is the nucleoside which modified base is designated as queuine which is an evolutionary ancient compound. The bacteria are unique in their ability to synthesize queuine and pass it on to plants and animals. For eukaryotes, queuine, recovered from digested food or gut microbiota, is converted to queuosine by placing it in the wobble position of several rRNAs, including aspartic acid, asparagine, histidine and tyrosine [7]. Queuine is transported into the cell through a rapidly saturating low $K_{\mathrm{m}}$ 
component and a slower-uptake high $K_{\mathrm{m}}$ component, and both cytosol and mitochondrial rRNA are modified by the queuine-insertase enzyme. Inside the cells, RNA polymerase III is used for transcription of cytosolic tRNA in the nucleus, while mitochondrial-directed RNA polymerase is used for the transcription of mitochondrial tRNA in mitochondria. Queuine base is incorporated into cytosolic and mitochondrial tRNAs on the mitochondrial membrane where co-localize with queuine-insertase complex which including QTRT1 and queuine-tRNA ribosyltransferase domain containing 1 (QTRT2 or QTRTD1) subunits [5]. In humans, queuine produced by the gut microbiome is the substrate of the heterodimeric enzyme of QTRT1/QTRT2 for tRNA modification, QTRT1 is the catalytic subunit that is conserved from bacteria to humans [8]. Thus, Q modification in body cells depends on intestinal microbiome. tRNA Q-modifications occur specifically in 4 cellular tRNAs at the wobble anticodon position that is directly involved in decoding of the genetic information $[9,10]$. tRNA Q-modifications also affect the cellular small RNA pools that can modulate cell physiology through RNA-protein interactions [8].

In this study, we aim to (i) examine microbiome dependency and tRNA Q-modification on breast tumor growth and (ii) identify the mechanisms of tRNA Q-modification dependent cellular phenotypes and on gene expression in vitro and in vivo. We generated single clones of complete QTRT1-KO in human breast cancer MCF7 cells. These clones were used for in vitro cells and in vivo mouse tumor studies. We identify the functions of the QTRT1 gene in regulating critical genes in cell proliferation, tight junction, and migration in human breast cancer cells and in the breast tumor mouse models. Importantly, we also find evidence that microbiome is involved in the breast cancer development in vivo. Our results demonstrate that at the cellular and molecular level, altered tRNA Q-modifications can play a critical role in breast cancer.

\section{Results}

\section{Establishment of QTRT1 knockout (KO) clone in breast cancer cell line}

To investigate the effects of QTRT1 on breast cancer tumor growth, we use the Double Nickase Plasmid to suppress the expression of QTRT1 in MCF7 cells. The plasmid encodes a D10A mutated Cas9 nuclease and a unique, target-specific 20-nt guide RNA (gRNA), which has greater specificity of gene knockout than the CRISPR/Cas9 KO plasmid counterpart (Figure 1a). After plasmid treatment and puromycin selection, the DNA and protein expression level of QTRT1 was below the threshold of detection in MCF7 breast cancer cells (Figure 1b, c). QTRT1-special amplicon upon PCR amplification of QTRT1-KO cells was absent (Figure 1b), which was further confirmed by genomic sequencing of the PCR products (Figure S1). The reduced QTRT1 expression in the knockout clone was persistent in different passages of cells, indicating that we established stable QTRT1-KO in human MCF7 cells. Knockdown of QTRT1 was also established in the MDA-MB-231 breast cancer cells (Figure S2 a, b). 


\section{Knockout of QTRT1 suppressed cell proliferation and migration}

After establishing the QTRT1-KO single clone in MCF7 cells, we examined cell proliferation by the MTT assay, which assesses viable cell metabolic activity. We found that cell proliferation was significantly reduced in QTRT1-KO MCF7 cells $(P<0.01)$ (Figure 1d) compared with its parental cells. The suppressed cell proliferation was further confirmed with significantly decreased expression of markers of cell proliferation PCNA and Ki67 in QTRT1-KO MCF7 cells (Figure 1e, f).

The adherent cells have the ability to migrate and heal the wound, like the re-epithelialization of the skin scratch. To investigate the role of QTRT1 in the migration of breast cancer cells, we investigated the wound healing ability of these cells. We found that cells with suppressed QTRT1 expression healed the wound area significantly slower than that of the WT cells (Figure 2a, b). QTRT1 knockout in MCF7 cells significantly reduced cell migration at all the timepoints we investigated for post wound healing (Figure $2 a, b)$. Three days post wounding the cells, the WT MCF7 cells healed around $20 \%$ of the wound area, whereas the QTRT1-KO cells healed less than $10 \%$ of the wound area $(P<0.05)$. After 9 days, WT cells healed $100 \%$ of the wound area compared with around $40 \%$ in QTRT1-KO MCF7 cells $(P<0.01)$ (Figure 2a, b).

The suppression of cell proliferation and migration of QTRT1 deletion were confirmed with the knockdown of QTRT1 in MDA-MB-231 breast cancer cells (Figure S2 c, d, e, f, g).

\section{Knockout of QTRT1 altered cell adhesion and tight junctions}

Cell adhesion plays an essential role in regulation of fundamental cellular process, such as cell proliferation and migration [11]. To elucidate the mechanism of how suppressed QTRT1 expression leads to decreased breast cancer cell proliferation and migration, we investigated the expression of cell adhesion protein markers such as $\beta$-catenin and E-cadherin. E-cadherin is known as an anti-cancer protein. We found that knockout of QTRT1 significantly increased expression of E-cadherin and $\beta$-catenin and in the QTRT1-KO MCF7 cells, compared with the WT cells (Figure 2c).

Tight junction proteins are the key players in epithelial barrier function. Claudin- 5 is an important tight junction protein, which is highly expression in breast cancer patient with high-risk metastasis and reoccurrence [12]. We found that QTRT1-KO MCF7 cells expressed significantly reduced the Claudin-5 at the protein level (Figure 2c). The increased expression of $\beta$-catenin and E-cadherin was further confirmed in QTRT1-KO MCF7 cells compared with wildtype cells using immunofluorescence staining (Figure 2d). After QTRT1-KO, the membrane $\beta$-catenin level was enhanced, suggesting the increased cell adhesion among cells. These data indicated an important role of QTRT1 in regulating the cell junction pathways.

The alteration of $\beta$-catenin and Claudin-5 was confirmed using the WT and QTRT1-KD MDA-MB-231 breast cancer cells (Figure $\mathbf{S} \mathbf{h}$ ). 


\section{Knockout of QTRT1 decreased tumor growth and altered TJ regulators in vivo}

The changes of breast cancer cells in vitro have been replicated using direct orthotopic or heterotopic injection of cells into the mouse [13-17]. To investigate the impact of QTRT1 knockout on tumor outgrowth, the xenograft nude mouse model ( $\mathrm{n}=20$ per group) was established by injecting QTRT1-KO and WT breast cancer cells. We found that the tumor number, volume and weight were significantly reduced in mice injected with QTRT1-KO MCF7 cells $(20 / 20)$ compared to the WT cells $(20 / 20)(P<0.01)$ (Figure 3a). The BrdU index (number of cell stained with BrdU / number of total cells) was significantly lower in mice injected with QTRT1-KO cells than that of WT cells $(P<0.01)$ (Figure $3 \mathbf{b})$, suggesting less cell growth in the QTRT1-KO cells in vivo. The expression of PCNA, another important proliferation regulator of cells, was also significantly decreased in tumors derived from QTRT1-KO MCF7 cells, compared with WT cells (Figure $3 c$, d).

As TJ proteins regulate several key signaling pathways in cancer process and development [18], we tested the markers of junction proteins in the tumors. Western blot analysis showed that the QTRT1-KO-induced tumors had significantly increased E-cadherin and $\beta$-catenin, but ablated expression levels of Claudin- 5 (Figure 3e). Immunofluorescence staining further showed increased $\beta$-catenin in tumors from QTRT1-KO injected mice, compared to that in WT MCF7 breast cancer cells (Figure $3 f$ ).

The suppression of tumor growth and alteration of tight junction regulators in the tumors were also found in the QTRT1 knockdown MDA-MB-231 cells using the xenograft nude mouse model (Figure S3).

\section{Bacteria were detected in tumors from the nude mice}

Two probes EUB338 and Bfi826 were used for detecting total bacteria and Butyrivibrio fibrisolvens-related clones which are gastrointestinal microbiome and ubiquitously present in the gastrointestinal tract of human and animals, respectively. The fluorescence in situ hybridization with probe EUB338 (target for total bacteria) and Bfi826 (target for Butyrivibrio fibrisolvens-related clones) highlighted the bacteria replication in the tumors from the nude mice. The relative concentration of bacteria in tumor induced from WT cells was significantly higher than that of QTRT1-KO cells using both EUB338 and Bfi826 probes $(P<0.01$ and $P<0.05$, respectively) (Figure 4a, b). The bacteria existence in the tumors were confirmed with the tumor samples from the mice challenged with QTRT1-KD and WT MDA-MB-231 cells (Figure S4 a).

\section{Altered gut microbiome in the nude mice injected with WT MCF7 cells}


We further investigated whether gut microbiomes have changed in the nude mice injected with WT MCF7 cells or QTRT1-KO cells.

The Kruskal-Wallis test showed that the Chao 1 richness in MCF7 Species level between WT and KO groups was statistically significant $(P=0.0112)$. KO group increased Chao 1 measure values from before treatment with mean (SD): 162.1(30.2), median: 151.3 to post treatment with mean (SD): 203.8(27.2), median: 209.8. The FDR adjusted P-value with the Benjamini-Hochberg method is 0.0106 . WT group also increased Chao 1 measure values from before treatment with mean (SD): 170.2(28.1), median: 160.9 to post treatment with mean (SD): 181.1(20.9), median: 179.7. However, the increase is not statistically significant (PDR $=0.3153)$. The Kruskal-Wallis test showed that the Shannon diversity in MCF7 Species level between WT and KO groups before and post treatment was also statistically significant $(P=$ 0.0002). KO group decreased Shannon measure values from before treatment with mean (SD): 2.5 (0.1), median: 2.5 to post treatment with mean (SD): 2.1(0.3), median: 2.1. The FDR adjusted P-value using the Benjamini-Hochberg method is 0.0028 . WT group also decreased Shannon measure values from before treatment with mean (SD): 2.4 (0.2), median: 2.4 to post treatment with mean (SD): 2.1(0.2), median: $2.1(\mathrm{FDR}=0.0099)$. However, comparing to $\mathrm{KO}$ group, WT group has less decrease.

Moreover, the bacterial 16s rRNA gene sequencing also showed that the intestinal microbial similarity of xenograft mice was different between the mice post-injection of WT and QTRT1-KO cells (Figure 4c-e; Figure S2). The breast cancer cell injected groups have significant different variations between WT MCF7 and QTRT1-KO groups ( $P=0.0028)$ using the variance homogeneity test (Figure 4c). ANOSIM further confirmed that the compositional dissimilarity of post-injection of WT MCF7 cells was significantly decreased compared with that of before-injection, but not of QTRT1-KO cells ( $P=0.001$ ) (Figure 4c). The exploratory analysis by principal coordinates analysis ( $\mathrm{PCOA}$ ) showed that before injection, the ranges of the Bray-Curtis dissimilarities between WT and KO mice were overlapped, however, the ranges of dissimilarities of microbiome were increased in mice post injection (Figure 4d). PERMANOVA test showed that the groups account for $76 \%$ variations of the Bray-Curtis dissimilarities $(P=0.01)$ (Figure $4 d$ ) which verified the ANOSIM results which maybe potentially explained by group-level differences. Several core bacteria were markedly changed in mice post cell injection, such as Lachnospiraceae, Lactobacillus and Alistipes (Figure 4e; Figure S5-6). The butyrate-producing genera Lachnospiraceae and Ruminococcaceae which may enhance producing butyric acid to protect the body from colon cancer [19] and triple negative breast cancer (TNBC) [20], was found suppressed in the mouse model post breast cancer cells injection. The proportions of Lactobacillus, one of the common colonic microbiota, are frequently correlated with intestinal diseases, like Crohn's Disease (CD), obesity, type 2 diabetes and cancer [21], and breast cancer tumor development [22], were found decreased as well. In the meantime, we found the abundance change of Alistipes, which may has disease-promoting activities with gut diseases, and relatively high incidence in the nipple aspirate fluid from people with breast cancer [23-25].

These microbial alteration was confirmed by real-time PCR with primers target for 16S rRNA gene of Lactobacillus, Lachnospiraceae and Butyrivibrio fibrisolvens, as described before [26-28] (Figure S6) in the intestinal feces from the MCF7 injected mice. The relative abundance of bacteria Lachnospiraceae 
was significantly altered between the compared groups ( $p<0.05)$ (Figure S6). The $16 \mathrm{~S}$ rRNA gene sequencing was also performed with the fecal samples from the MDA-MB-231 challenged mice (Figure S4 b, c, d).

\section{Inflammation enhanced the bacteria in tumors of nude mice}

Based on the results above we hypothesized that inflammation may lead to the increased bacteria population in tumors. The CD68-postive and CD-11b-positive inflammation-related cells were immunostained in the tumors from nude mice injected both WT and QTRT1-KO breast cancer cells which indicated immune response inside the tumors (Figure 5a). The number of CD68-postive and CD11bpostive cells in the tumors from the mice injected WT MCF7 cells was significantly higher than that of QTRT1-KO cells $(P<0.05)$ (Figure 5a). We further investigated the cytokines and chemokines level in the plasma of the mouse model. MCP-3, the chemotactic gradients implicated in the process of inflammation, and Interleukin 6 (IL-6), one of the interleukin acts as both pro-inflammatory cytokine and anti-inflammatory myokine, were found markedly increased in the mice injected with WT cells, compared with that of QTRT1-KO cells (Figure 5b). We did not found significant change of IL-10 or TNF-a (Figure $5 b)$.

\section{Disrupted tight junctions in the colon of mice injected with WT MCF7 cells}

Tight Junction (TJ) proteins, including claudins (like Claudin 2, Claudin 4) and Zona occludens (like ZO-1, ZO-2), have an important role in maintaining the integrity of the gut epithelium. Dysfunction of TJs, including changes in protein distribution and expression level, could result in increased permeability and chronic inflammation [18]. Leaky gut is known to lead to dysbiosis and enhance inflammation [29, 30]. We examined the intestinal tight junctions of the nude mice that were injected with WT or QTRT1-KO MCF7 cells. We found that the expression of the tight junction protein ZO-1 was reduced in the colon of nude mice injected with WT cells (Figure 6a). The structure disruption of ZO-1 was visualized in the colon of WT cell injected mice, whereas the ZO-1 distribution is intact in the colon of mice injected with QTRT1$\mathrm{KO}$ cell (Figure 6a). Claudin 2 is considered a leaky protein which increases the intestinal permeability intestinal permeability [30,31]. Interestingly, we found the significantly increased Claudin 2 in the colon of WT cell injected mice, suggesting the enhanced leaky gut (Figure $6 \mathbf{b}$ ). In contrast, the colon of mice injected with QTRT1-KO cell showed significantly reduced Claudin 2 (Figure 6b).

\section{Discussion}

In the current study, we have demonstrated that QTRT1 plays a crucial role in breast cancer cell proliferation and growth both in vitro and in vivo. The proliferation and collective migration of QTRT1-KO MCF7 breast cancer cells were markedly suppressed, compared with its parent cells. Meanwhile, QTRT1 
knockout leaded to changes in the expression of E-cadherin, $\beta$-catenin and claudin-5, which are important cell junction proteins and facilitate cells adhesin formation. These differences were further confirmed in the xenograft nude mouse model in vivo. Moreover, we found elevated levels of microbiome in the tumors from WT-MCF7 cell injected mice. Increased inflammation and disrupted tight junctions in the colon of mice injected with WT MCF7 cells may contribute to accelerated breast cancer development in vivo (Figure 6c). Furthermore, the deletion of QTRT1 suppressed cell proliferation, migration and alteration of TJs in vitro, and tumor growth, altered TJs regulators and microbial alteration of tumor and intestine in vivo, were also confirmed using the wildtype and QTRT1-KD MDA-MB-231 breast cell line, which further highlighted the importance of QTRT1 in breast cancer development and progress.

The breast is not sterile but contains a diverse and unique bacterial community which is distinct from that of other body sites [34]. In vivo, we investigated the inflammatory and bacterial profile in the tumor in situ and the organismic system of the mouse. Here, we detected the existence of bacteria in the tumors grown in the xenograft nude mice injected QTRT1-KO and WT MCF7 cells. Meanwhile, we found that the enriched bacteria which in tumor from WT MCF7 cells were correlated with increased inflammation inside the tumors. These data suggest bacterial replication in the tumor that leads to changed microenvironment. Although it is still unclear whether the microbial differences are the consequences or causes of breast cancer or other breast diseases, the changes in the composition of breast microbiota could contribute to disease development and progression [34]. Similarly, our findings indicate that the changed microenvironment of the tumor may contribute to the tumor growth, which is another important factor other than QTRT1 knockout.

The source of the bacteria in tumors may shed light on breast tumor development. B. fibrisolvens belongs to the genus Butyrivibrio and is part of the gastrointestinal microbiome of mammals and ubiquitously present in the gastrointestinal tract of many animals, including human and mouse [35]. These bacteria inside the tumors may be translocated from the intestine. Indeed, the intestinal lumen and outer mucus layer are the location of overwhelming majority of gut bacteria, but it seems reasonable to envisage that a very small but significant minority of bacteria could occasionally breach the intestinal epithelium and quickly arrive at other organs or tissues, especially in the nude mice which are immune-deficient in our study. Recently, more and more data support the transfer of intestinal bacterial products and bacteria translocation directly or indirectly in some diseases, such as liver disease [36, 37]. This hypothesis may be supported by the redistribution of ZO-1 and increased leaky protein Claudin 2 in the colon of the nude mice, which is related with the intestinal permeability [38]. Tight junction proteins are the key players in the epithelial barrier function in inflammatory bowel disease [38-42]. Furthermore, the gut microbiome community of mouse model markedly changed after breast cancer cell injection, compared with previous ones based on $16 \mathrm{~S}$ ribosomal RNA gene amplification data. Also, taxonomic richness and diversity differences were found between the mice injected with WT and KO cells. The intestinal dysbiosis, especially in the mice injected with WT cell, should further enhance the disfunctions in intestine. Therefore, the alteration of tight junction proteins and microbiome components in the intestine should enhance the bacterial translocation by regulating intestinal bacterial interactions with the mucosal surface, which may support the bacterial translocation from the intestine to other locations or the 
tumors. Increased cytokine and chemokine in the plasma of the mice injected with WT MCF7 cells also support the idea that inflammation and leaky gut promote dysbiosis [43] and bacterial translocation.

Bacteria which enhance producing butyric acid are known to protect the body from colon cancer [19] and triple negative breast cancer (TNBC) [20], Our data on the reduced butyrate-producing genera

Lachnospiraceae and Ruminococcaceae in the mice with breast cancer cells support the role of intestinal dysbiosis in promoting breast cancer. Intestinal Lactobacillus are frequently correlated with intestinal diseases, like Crohn's Disease (CD), obesity, type 2 diabetes and cancer [21], and breast cancer tumor development [22]. We also found Lactobacillus decreased in mice with breast cancer cells. In the meantime, we found the abundance change of Alistipes, which may promote digestive diseases, and relatively high incidence in the nipple aspirate fluid from people with breast cancer [23-25]. The potential origin of part of breast tissue microbiome may be translocated from the gastrointestinal tract in addition to the skin via the nipple-areolar orifices [44]. Our data suggest the potential translocation of gut microbiome in breast tumors.

Beta-catenin is a multi-functional protein involved in cell adhesion, cell signaling and regulation of gene transcription depending on its intracellular localization [32]. In our study, QTRT1 knockout in MCF7 cells could significantly increase the expression of $\beta$-catenin and change its location from inside the cells to the membrane, which participates in the formation, maintenance and function of adherence junctions by linking cadherin to the actin cytoskeleton [32]. The E-cadherin, one of the classical cadherins which play crucial role in the cell integrity and polarity, was also found upregulated in the QTRT1-KO cells. Thus, both the changes of E-cadherin and $\beta$-catenin impact on the cell adhesion, combination and polarity, and further reduce the proliferation and migration of MCF7 cells after QTRT1 knockout. Claudins are major adhesion molecules in tight junctions and are strongly expressed in various cancers [33], and Claudin-5 is highly expressed in breast cancer patients [13]. Interestingly, we found the downregulation of Claudin-5 in QTRT1-KO MCF7 cells. Suppressed cell proliferation in QTRT1 knockout contributed to marked reduction of the tumor growth, number, volume, and weight.

The knockout of QTRT1 in MCF7 cells could impact on the cell proliferation ability which influences the microenvironment of the tumors, which could feedback to affect the bacterial multiplication in the tumor. Moreover, the inflammation investigated in the tumors with related biomarkers (CD68 and CD11b) may be related with the bacterial growth in the tumors and microenvironment modification. More studies need to be done to investigate the relationship between microbiome in tumor and tumor growth, and the source of these microorganisms. Another hypothesis was that the bacteria found in the tumors may be transferred from the skin which touches the surround feces in the mouse cage. Further studies are also needed to understand the mechanisms of how QTRT1 interacts within TJ proteins to influence the cell proliferation and tumor growth.

\section{Conclusions}


Conclusively, we found microbiome and microenvironmental alteration in tumors and intestine which may be related. Lacking microbiome-dependent tRNA Q-modifications suppresses breast tumor growth. we have demonstrated the important functions of QTRT1 in regulating cancer cells. Knockout of QTRT1 could significantly suppress the proliferation and migration ability of breast cancer cells. The knockout of QTRT1 resulted in the loss of aggressiveness of cancer cells both in vitro and in vivo. Our findings provide new insights into manipulating QTRT1 as a potential gene in controlling tumor growth and development.

\section{Methods}

\section{Regents and cell lines}

The monoclonal antibody to human QTRT1 was purchased from Santa Cruz Biotechnology (Dallas, TX). Monoclonal antibody of $\beta$-catenin and E-cadherin from BD Transduction (San Jose, CA), and $\beta$-actin were purchased from Sigma-Aldrich (St. Louis, MO). Monoclonal antibody of PCNA and CD11b (Integrin aM) were purchased from Santa Cruz Biotechnology (Dallas, TX). Claudin 5 and Claudin 2 mouse monoclonal antibody and ZO-1 rabbit monoclonal antibody were purchased from Thermo Fisher Scientific (Rockford, IL). CD68 rabbit polyclonal antibody was purchased from Abcam (Cambridge, MA). All chemicals were purchased from Sigma-Aldrich unless otherwise stated. The breast cancer cell lines, including MCF7 and MDA-MB-231, were provided by Dr. Tao Pan's lab at University of Chicago.

\section{Transfection and selection}

The highly aggressive breast cancer cell line human MCF7 cells were cultured in a 6-well tissue culture plate in antibiotic-free MCF7 cells growth medium (Eagle's Minimum Essential Medium with 10\% FBS, $10 \mathrm{ug} / \mathrm{ml}$ bovine insulin, $10 \mathrm{nM} \beta$-estradiol) (Invitrogen, Carlsbad, CA) to 70-80\% confluence. Cells were transfected with $2 \mu \mathrm{g}$ of QTRT1 Double Nickase Plasmid (sc-413456-NIC), 10 $\mathrm{ll}$ LTX Lipofectamine and 2.5ul PLUS Reagent (Invitrogen) per well (manufacturer's protocol). QTRT1 Double Nickase Plasmidderived puromycin-resistance gene was used for positive selection of transfected cells. Cells were selected with $1 \mu \mathrm{g} / \mathrm{ml}$ puromycin for 5 days when further cell death was not observed. After selection, cells were collected, and serial diluted onto four 96 -well plates. Single cells were expanded to obtain individual clones. The culture of MDA-MB-231 cells was as described before[45], and transfection and selection were as described above.

\section{Clone validation}

Individual clones after expansion to 6-well plate format were collected for clone validation. Cells were lysed in CelLytic ${ }^{T M} M$, Lysis Reagent (Sigma-Aldrich) with protease inhibitor cocktail (ThermoFisher) overnight at $4^{\circ} \mathrm{C}$. The lysate was centrifuged at $16,000 \mathrm{~g}$ for $30 \mathrm{~min}$ and $10 \mu \mathrm{g}$ of supernatant was fractioned on 4-20\% SDS PAGE gels, transferred to nitrocellulose, and screened by Western Blot with QTRT1 Antibody (Santa Cruz; sc-398918). Antibody (Santa Cruz; sc-398918) is specific for amino acids 
111-136, exon 3 and a 5 ' fragment of exon 4: QTRT1Genomic DNA was isolated from edited clones and nonedited MCF7 control cells with DNAzol method (ThermoFisher) and tested with PCR using QTRT1specific primers in exon 1 (F1: $5^{\prime}$ end of the exon1: GGCGGGAGCAGCTACCCA) and intron downstream of exon 3 (Rev1_3: intron downstream of the exon 3: CCCGGCCTCAAGTGATCTTC). The clone validation of MD-MB-231 cells was performed as described above.

\section{Cell Proliferation Assay}

The WT and QTRT1 KO MCF7 cells were plated in a density of $1 \times 10^{4}$ cells per well in triplicate. After two days of culture, the proliferation was evaluated by MTT Cell Proliferation Assay Kit (Thermo Fisher Scientific, Rockford, IL) according to the product's instructions [45]. Triplicate wells were counted for each time point and the whole experiment was repeated three times. The evaluation of proliferation of WT MDA-MB-231 and QTRT1-KD (knockdown) MDA-MB-231 cells was performed as described above using MTT Cell Proliferation Assay Kit.

\section{Wound Healing Assay}

Both wild type (WT) and QTRT1-KO of MCF7 were plated on dishes (MatTek, Ashland, MA) and cultured in humidified chambers with $5 \% \mathrm{CO}_{2}$ at $37^{\circ} \mathrm{C}$. When Cells were grown to confluency, the wounds were scratched and analyzed as described before [45]. Migration of cells into wounded areas was captured at per day. The values are the means of three independent wound fields from three independent experiments $(n=3)$. As described above, the wound healing assay was also performed on the WT and QTRT1-KD MDA-MB-231 cells.

\section{In vivo nude mice model}

The 8-week old, specific-pathogen-free, female BALB/c Nude mice $(n=40)$ were purchased from Charles River Laboratories (Wilmington, MA). Animals were housed in Biologic Resources Laboratory (BRL) at University of Illinois at Chicago (UIC) and utilized in accordance with the UIC Animal Care Committee (ACC) and Office of Animal Care and Institutional Biosafety (OACIB) guidelines. The same and consistent diet was provided to the animals throughout the experiment. The animals were separated as two groups including mice for MCF7-WT cell injection $(n=20)$ and mice for QTRT1-KO MCF7 cell injection $(n=20)$. The xenograft model was established by subcutaneous bilaterally injecting with $1.2 \times 10^{6}$ cells in $200 \mu \mathrm{l}$ of 50:50 Matrigel / PBS (phosphate buffered saline) into the hind flank [46]. At 60 days post-tumor challenge, tumors and samples were harvested from the animals which were euthanased by IP injection of sodium pentobarbital (100mg per kg body weight) followed by cervical dislocation. The weight of the tumors was scaled, and the tumor volume $(V)$ was calculated with caliper measurements using formulas $V=\left(W^{2} \times L\right) / 2$ [47]. BrdU staining was performed as previously described [48]. Protein expression in tumor tissues was detected by immunofluorescence staining and western blot as described below. 
Wildtype and QTRT1-KD MDA-MB-231 cells $\left(7.5 \times 10^{6}\right)$ were also performed using the BALB/c Nude mice $(n=40)$ as described above, except that the tumors and samples were harvested at 30-day post cell injection.

\section{Western blot analysis}

Breast tissue was lysed in stocked lysis buffer [45]. Cultured cells were rinsed twice in ice-cold Hanks' balanced salt solution (Sigma-Aldrich, Saint Louis, MO) and lysed in protein loading buffer then followed by sonication (Branson Sonifier, Danbury, CT) and centrifugation [45]. The target proteins were detected by special primary antibody $(1: 1,000)$ followed by secondary antibody conjugated to horseradish peroxidase at 1:5,000 dilution. The blots were visualized by ECL chemiluminescence (Thermo Scientific, Rockford, IL). All experiments were performed 3-5 times. Western blot bands were quantified using image analyzer (ImageJ, NIH, Bethesda, MD).

\section{Immunofluorescence and confocal Imaging}

Wildtype and knockout/knockdown cells were plated on fibronectin-coated glass coverslips and cultured with medium described above to monolayer in humidified chambers with $5 \% \mathrm{CO}_{2}$ at $37^{\circ} \mathrm{C}$. The cells were immunostained and imaged as described before [45]. All experiments were performed multiple times using independent biological replicates.

Fresh tumors were fixed in $10 \%$ neutral buffered formalin followed by paraffin embedding. For immunofluorescence staining [49], slides were incubated in 5\% BSA (Bovine Serum Albumin) with 0.1\% goat serum in PBS for 1 hour at room temperature to reduce nonspecific background. The samples were incubated overnight at $4^{\circ} \mathrm{C}$ with primary antibody at 1:100 dilution. The sections were then incubated with secondary antibodies and DAPI for 1 hour at room temperature, and examined with confocal microscope as described before [50].

For fluorescence in situ hybridization [4] staining, fresh tumor tissue was fixed with $10 \%$ neutral buffered formalin and embedded in paraffin. The sections were treated successively with $0.2 \mathrm{M} \mathrm{HCl}, 1 \mathrm{M} \mathrm{NaSCN}$ and $4 \%$ pepsin, then hybridized with the EUB338 probe (5'-GCTGCCTCCCGTAGGAGT-3') for all bacteria and Bfi826 (5'-ATGGCACCCAACACCTAG-3') for Butyrivibrio fibrisolvens-related clones[51] in hybridization buffer $\left(0.9 \mathrm{M} \mathrm{NaCl}, 0.02 \mathrm{M}\right.$ Tris- $\mathrm{HCl}, \mathrm{pH} 7.6,0.01 \%$ sodium dodecyl sulfate) at $37^{\circ} \mathrm{C}$ overnight. Slides were then incubated for $10 \mathrm{~min}$ with FISH washing buffer $(0.3 \mathrm{M} \mathrm{NaCl}, 0.03 \mathrm{M}$ Trisodium citrate) preheated to $45^{\circ} \mathrm{C}$. Slides were imaged using confocal microscope as described above. All experiments were performed 3-5 times.

\section{Immunohistochemistry}


Tissues were fixed in 10\% neutral buffered formaldehyde for 2 hours, transferred into $70 \%$ ethanol, and processed the next day by standard techniques. Immunohistochemistry for inflammation cells was performed on paraffin-embedded sections $(4 \mu \mathrm{m})$ of tumors. Briefly, the paraffin sections were baked in an oven at $56^{\circ} \mathrm{C}$ for 30 minutes. The sides were deparaffinized and rehydrated in xylene, followed by graded ethanol washes at room temperature. Antigen retrieval was achieved by boiling the slides in the microwave oven with $0.01 \mathrm{M}, \mathrm{PH} 6.0$ sodium citrate buffer. Sides were then incubated in hydrogen peroxide ( $3 \% \mathrm{H}_{2} \mathrm{O}_{2}$ in PBS) for 10 minutes at room temperature, followed by incubation in $5 \%$ fetal bovine serum/PBS for 1 hour. The inflammation cells were stained with polyclonal CD68 antibody (abcam, Cambridge, MA) and monoclonal CD11b antibody (Santa Cruz, Dallas, TX).

\section{Luminex immunoassays}

The 26 cytokines and chemokines of plasma samples were analyzed using the mouse cytokine and

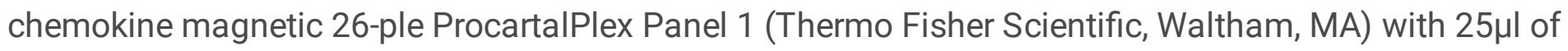
samples according to the manufacturer's instructions using a 2 hours incubation at room temperature. Samples were read on a MAGPIX ${ }^{\mathrm{TM}}$ system platform (Millipore Sigma, Burlington, MA).

\section{Microbial sampling and sequencing}

The day before the cell injection, fresh feces were collected directly from the mouse into the sterile tubes. At the end of the experiment, fresh fecal samples were isolated from the colon and placed into the specially prepared sterile tubes. The samples were kept at low temperature with dry ice and were sent to the UIC RRC (University of Illinois at Chicago Research Resources Center) for genomic sequencing. The DNAs of samples were extracted using DNeasy Power Fecal Kit (Qiagen, Hilden, Germany) based on manufacturer's instructions with a slight modification. The samples were heated at $65^{\circ} \mathrm{C}$ for $10 \mathrm{~min}$ before homogenizing with FastPre-24 $5 \mathrm{G}$ bead-beating device (MP Biomedicals, Solon, $\mathrm{OH}$ ) at $6 \mathrm{~m} / \mathrm{s}$ for 40 seconds. The workflow for preparing samples for next-generation amplicon sequencing contains two independent PCR steps. The first stage PCR amplification was performed using primers containing CS1 and CS2 linkers (CS1_341F: 5'-ACACTGACGACATGGTTCTACAGTGCCAGCMGCCGCGGTAA-3'; CS2_806R: 5'-TACGGTAGCAGAGACTTGGTCTGGACTACHVGGGTWTCTAAT-3') to the V3-V4 variable region of the 16S rRNA gene, while the second stage PCR amplification was performed on the first stage PCR products using the Fluidigm Access Array barcoded primers. The 16S rRNA gene metagenomic sequencing was performed using MiSeq according to the Illumina protocol.

\section{Sequencing bioinformatics}

All possible raw paired-end reads were evaluated and merged using the PEAR (Paired-End reAd mergeR) software (http://www.exelixis-lab.org/web/software/pear.) [52]. Ambiguous nucleotides were trimmed from the ends and reads with internal ambiguous nucleotides were discarded. Filter-passed reads were 
used for further analysis after trimming off both primer and adapter sequences based on quality threshold $(P=0.01)$ and length (minimum length=225). Chimeric sequences were identified using the USEARCH (http://www.drive5.com/usearch) algorithm as compared with the reference database silva_132_16S.97 [53]. Then, operational taxonomic unit (OTU) clusters were generated using the UCLUST (http://www.drive5.com) method with a threshold of $97 \%$ sequence similarity. Taxonomic annotation of each OTU was made via searching by similarity against the references which were identified using the USEARCH (http://www.drive5.com/usearch) algorithm as compared with a reference database [54].

Initially, 1,314,538 reads were assembled from the source sequencing data. After trimming, the number of reads were diminished to $1,290,241$, and then after chimera checking reduced to $1,211,340$ which was used as the operational OTUs in the alignment. The OTUs were ranged from 54,043 to 65,772 for the individual sample, with a mean of 60,567. The taxonomic assignments of the microbiomes of the studied samples were obtained with the OTU data. On the phylum level, Bacteroidetes and Firmicutes accounted for the major part of the microbial population in all samples (96.4\%-99.4\%).

\section{Statistical analysis}

Data shown in the bar figures were the average values from at least three independent experiments with the Mean \pm SD. All statistical tests were two-sides. It was considered statistically significant with $P$-value $<0.05$. One, two and three asterisks on the bars in the figures represent $P$-value $<0.05,<0.01$, and $<0.001$, respectively. To investigate the dynamic effects of QTRT1-KO/KD on cell migration in breast cancer cells, the group effects from 1 to 9 days (Figure 2a, Figure S2f) were tested using generalized linear mixed models.

The alpha and beta diversity indices including Shannon diversity, Chao1 richness and Bray-Curtis dissimilarity were calculated. The "core" bacteria were defined as the set of species shared by (almost) all individuals with $0.1 \%$ relative abundance in more than $50 \%$ of the samples. Bar plot and heatmap were used to visualize the identified core bacteria. Principal coordinate analysis (PCoA) plots were used for the visualization of Bray-Curtis dissimilarity [55]. To detect the differences of Bray-Curtis dissimilarity among groups, PERMANOVA was performed, followed by a variance homogeneity assumption testing to ensure the reliability of the PERMANOVA results. Then a nonparametric procedure analysis of similarity (ANOSIM) based on a permutation test was used for analyzing among-and-within group similarity. The latest version $\mathrm{R}$ and $\mathrm{R}$ packages of ampvis2, microbiome, phyloseq and vegan were used for microbiome data analyses[56, 57].

The statistical analyses were performed using SAS version 9.4 (SAS Institute, Inc. Cary, NC), GraphPad Prism 5 (GraphPad Software, Inc. La Jolla, CA).

\section{Declarations}




\section{Ethics approval and consent to participate}

Animals were housed in Biologic Resources Laboratory (BRL) at University of Illinois at Chicago (UIC) and utilized in accordance with the UIC Animal Care Committee (ACC) and Office of Animal Care and Institutional Biosafety (OACIB) guidelines.

\section{Consent for publication}

Not applicable.

\section{Availability of data and material}

The raw sequence data from $16 \mathrm{~S}$ rRNA gene amplicon sequencing were submitted to NCBI SRA under BioProject accession number PRJNA599383 (https://www.ncbi.nlm.nih.gov/sra/PRJNA599383).

\section{Competing interests}

The authors declare that they have no competing interests.

\section{Funding}

This project was supported by the National Institute of Diabetes and Digestive and Kidney Diseases [R01DK105118-01 and R01DK114126 to J.S.]; United States Department of Defense Congressionally Directed Medical Research Programs [BC160450 to J.S. and T.P.]; and the UIC Cancer Center funds to Jun Sun.

\section{Authors' contributions}

JS secured research funding, designed the study, analyzed data, and finalized the manuscript; JZ, RL and $Y Z$, performed experiments and collected and analyzed data in vitro and in vivo; ZM and TP generated the $\mathrm{KO}$ cells; JZ and JS drafted the article; YX provided support for the study design and statistic analysis. All authors were involved in writing the manuscript and reviewed and approved the final version.

\section{Acknowledgements}

The authors thank the University of Illinois at Chicago Sequencing Core (UICSQC) for library preparation and sequencing, and the University of Illinois at Chicago Core for Research Informatics (UICCRI) for basic processing of the raw data.

\section{References}

1. Honda S, Loher P, Shigematsu M, Palazzo JP, Suzuki R, Imoto I, Rigoutsos I, Kirino Y: Sex hormonedependent tRNA halves enhance cell proliferation in breast and prostate cancers. Proc Natl Acad Sci 
U S A. 2015; 112:E3816-25.

2. Guzman N, Agarwal K, Asthagiri D, Yu L, Saji M, Ringel MD, Paulaitis ME: Breast Cancer-Specific miR Signature Unique to Extracellular Vesicles Includes "microRNA-like" tRNA Fragments. Mol Cancer Res. 2015; 13:891-901.

3. Dhahbi JM, Spindler SR, Atamna H, Boffelli D, Martin DI: Deep Sequencing of Serum Small RNAs Identifies Patterns of 5 ' tRNA Half and YRNA Fragment Expression Associated with Breast Cancer. Biomark Cancer. 2014; 6:37-47.

4. Goodarzi H, Liu X, Nguyen HC, Zhang S, Fish L, Tavazoie SF: Endogenous tRNA-Derived Fragments Suppress Breast Cancer Progression via YBX1 Displacement. Cell. 2015; 161:790-802.

5. Fergus C, Barnes D, Alqasem MA, Kelly VP: The queuine micronutrient: charting a course from microbe to man. Nutrients. 2015; 7:2897-929.

6. Morris RC, Brown KG, Elliott MS: The effect of queuosine on tRNA structure and function. Journal of Biomolecular Structure and Dynamics. 1999; 16:757-74.

7. Ames BN: Prolonging healthy aging: Longevity vitamins and proteins. Proceedings of the National Academy of Sciences. 2018; 115:10836-44.

8. Wang X, Matuszek Z, Huang Y, Parisien M, Dai Q, Clark W, Schwartz MH, Pan T: Queuosine modification protects cognate tRNAs against ribonuclease cleavage. Rna. 2018; 24:1305-13.

9. Muller M, Legrand C, Tuorto F, Kelly VP, Atlasi Y, Lyko F, Ehrenhofer-Murray AE: Queuine links translational control in eukaryotes to a micronutrient from bacteria. Nucleic Acids Res. 2019; 47:3711-27.

10. Tuorto F, Legrand C, Cirzi C, Federico G, Liebers R, Muller M, Ehrenhofer-Murray AE, Dittmar G, Grone HJ, Lyko F: Queuosine-modified tRNAs confer nutritional control of protein translation. Embo j. 2018; 37.

11. Matsunaga T, lyoda T, Fukai F: Adhesion-dependent cell Regulation via Adhesion molecule, integrin: Therapeutic application of integrin activation-modulating factors. In Colloid and Interface Science in Pharmaceutical Research and Development. Elsevier; 2014: 243-60

12. Sugimoto H, Nagahara M, Bae Y, Nakagawa T, Ishikawa T, Sato T, Uetake H, Eishi Y, Sugihara K: Clinicopathologic relevance of claudin 5 expression in breast cancer. Am J Clin Pathol. 2015; 143:540-6.

13. Di Cello F, Shin J, Harbom K, Brayton C: Knockdown of HMGA1 inhibits human breast cancer cell growth and metastasis in immunodeficient mice. Biochem Biophys Res Commun. 2013; 434:70-4.

14. Ooms LM, Binge LC, Davies EM, Rahman P, Conway JR, Gurung R, Ferguson DT, Papa A, Fedele CG, Vieusseux JL, et al: The Inositol Polyphosphate 5-Phosphatase PIPP Regulates AKT1-Dependent Breast Cancer Growth and Metastasis. Cancer Cell. 2015; 28:155-69.

15. Stengel C, Newman SP, Leese MP, Thomas MP, Potter BV, Reed MJ, Purohit A, Foster PA: The In Vitro and In Vivo Activity of the Microtubule Disruptor STX140 Is Mediated by Hif-1 Alpha and CAIX Expression. Anticancer Res. 2015; 35:5249-61. 
16. Yano S, Takehara K, Miwa S, Kishimoto H, Tazawa H, Urata Y, Kagawa S, Bouvet M, Fujiwara T, Hoffman RM: In Vivo Isolation of a Highly-aggressive Variant of Triple-negative Human Breast Cancer MDA-MB-231 Using Serial Orthotopic Transplantation. Anticancer Res. 2016; 36:3817-20.

17. Khaled M, Belaaloui G, Jiang ZZ, Zhu X, Zhang LY: Antitumor effect of Deoxypodophyllotoxin on human breast cancer xenograft transplanted in BALB/c nude mice model. J Infect Chemother. 2016; 22:692-6.

18. Bhat AA, Uppada S, Achkar IW, Hashem S, Yadav SK, Shanmugakonar M, Al-Naemi HA, Haris M, Uddin S: Tight Junction Proteins and Signaling Pathways in Cancer and Inflammation: A Functional Crosstalk. Front Physiol. 2018; 9:1942.

19. Meehan CJ, Beiko RG: A phylogenomic view of ecological specialization in the Lachnospiraceae, a family of digestive tract-associated bacteria. Genome Biol Evol. 2014; 6:703-13.

20. Collard M, Austin N, Tallant A, Gallagher P: Muscadine Grape Extract Reduces Lung and Liver Metastasis in Mice with Triple Negative Breast Cancer in Association with Changes in the Gut Microbiome (P05-017-19). Curr Dev Nutr. 2019; 3.

21. Heeney DD, Gareau MG, Marco ML: Intestinal Lactobacillus in health and disease, a driver or just along for the ride? Curr Opin Biotechnol. 2018; 49:140-7.

22. Jafari S, Froushani SMA, Tokmachi A: Combined Extract of Heated 4T1 and a Heat-Killed Preparation of Lactobacillus Casei in a Mouse Model of Breast Cancer. Iran J Med Sci. 2017; 42:45764.

23. Mancabelli L, Milani C, Lugli GA, Turroni F, Cocconi D, van Sinderen D, Ventura M: Identification of universal gut microbial biomarkers of common human intestinal diseases by meta-analysis. FEMS Microbiol Ecol. 2017; 93.

24. Yang Y, Jobin C: Novel insights into microbiome in colitis and colorectal cancer. Curr Opin Gastroenterol. 2017; 33:422-7.

25. Chan AA, Bashir M, Rivas MN, Duvall K, Sieling PA, Pieber TR, Vaishampayan PA, Love SM, Lee DJ: Characterization of the microbiome of nipple aspirate fluid of breast cancer survivors. Sci Rep. 2016; 6:28061.

26. Frank JA, Reich Cl, Sharma S, Weisbaum JS, Wilson BA, Olsen GJ: Critical evaluation of two primers commonly used for amplification of bacterial 16S rRNA genes. Appl Environ Microbiol. 2008; 74:2461-70.

27. Kennedy NA, Walker AW, Berry SH, Duncan SH, Farquarson FM, Louis P, Thomson JM: The impact of different DNA extraction kits and laboratories upon the assessment of human gut microbiota composition by 16S rRNA gene sequencing. PloS one. 2014; 9:e88982.

28. Zhang Y-g, Wu S, Yi J, Xia Y, Jin D, Zhou J, Sun J: Target intestinal microbiota to alleviate disease progression in amyotrophic lateral sclerosis. Clinical therapeutics. 2017; 39:322-36.

29. Clapp M, Aurora N, Herrera L, Bhatia M, Wilen E, Wakefield S: Gut microbiota's effect on mental health: The gut-brain axis. Clin Pract. 2017; 7:987. 
30. Pan ZY, Yang Y, Pan H, Zhang J, Liu H, Yang Y, Huang G, Yin L, Huang J, Zhou WP: Lentivirusmediated TPD52L2 depletion inhibits the proliferation of liver cancer cells in vitro. Int J Clin Exp Med. 2015; 8:2334-41.

31. Zhang YG, Wu S, Xia Y, Sun J: Salmonella infection upregulates the leaky protein claudin-2 in intestinal epithelial cells. PLoS One. 2013; 8:e58606.

32. Terada N, Karim MR, Izawa T, Kuwamura M, Yamate J: Immunolocalization of beta-catenin, Ecadherin and N-cadherin in neonate and adult rat kidney. J Vet Med Sci. 2017; 79:1785-90.

33. Jia W, Lu R, Martin TA, Jiang WG: The role of claudin-5 in blood-brain barrier (BBB) and brain metastases (review). Mol Med Rep. 2014; 9:779-85.

34. Fernandez MF, Reina-Perez I, Astorga JM, Rodriguez-Carrillo A, Plaza-Diaz J, Fontana L: Breast Cancer and Its Relationship with the Microbiota. Int J Environ Res Public Health. 2018; 15.

35. Rodriguez Hernaez J, Ceron Cucchi ME, Cravero S, Martinez MC, Gonzalez S, Puebla A, Dopazo J, Farber M, Paniego N, Rivarola M: The first complete genomic structure of Butyrivibrio fibrisolvens and its chromid. Microb Genom. 2018; 4.

36. Chassaing B, Etienne-Mesmin L, Gewirtz AT: Microbiota-liver axis in hepatic disease. Hepatology. 2014; 59:328-39.

37. Ma HD, Wang YH, Chang C, Gershwin ME, Lian ZX: The intestinal microbiota and microenvironment in liver. Autoimmun Rev. 2015; 14:183-91.

38. Lee SH: Intestinal permeability regulation by tight junction: implication on inflammatory bowel diseases. Intest Res. 2015; 13:11-8.

39. Shang M, Sun J: Vitamin D/VDR, Probiotics, and Gastrointestinal Diseases. Curr Med Chem. 2017; 24:876-87.

40. Landy J, Ronde E, English N, Clark SK, Hart AL, Knight SC, Ciclitira PJ, Al-Hassi HO: Tight junctions in inflammatory bowel diseases and inflammatory bowel disease associated colorectal cancer. World $\mathrm{J}$ Gastroenterol. 2016; 22:3117-26.

41. Sun J, Kato I: Gut microbiota, inflammation and colorectal cancer. Genes Dis. 2016; 3:130-43.

42. Lechuga S, Ivanov Al: Disruption of the epithelial barrier during intestinal inflammation: Quest for new molecules and mechanisms. Biochim Biophys Acta Mol Cell Res. 2017; 1864:1183-94.

43. Rosean CB, Bostic RR, Ferey JC, Feng T-Y, Azar FN, Tung KS, Dozmorov MG, Smirnova E, Bos PD, Rutkowski MR: Pre-existing commensal dysbiosis is a host-intrinsic regulator of tissue inflammation and tumor cell dissemination in hormone receptor-positive breast cancer. Cancer research. 2019:canres. 3464.2018.

44. Hieken TJ, Chen J, Hoskin TL, Walther-Antonio M, Johnson S, Ramaker S, Xiao J, Radisky DC, Knutson KL, Kalari KR, et al: The Microbiome of Aseptically Collected Human Breast Tissue in Benign and Malignant Disease. Sci Rep. 2016; 6:30751.

45. Zhang J, Li K, Zhang Y, Lu R, Wu S, Tang J, Xia Y, Sun J: Deletion of sorting nexin 27 suppresses proliferation in highly aggressive breast cancer MDA-MB-231 cells in vitro and in vivo. BMC Cancer. 
2019; 19:555.

46. Kocaturk B, Versteeg HH: Orthotopic injection of breast cancer cells into the mammary fat pad of mice to study tumor growth. J Vis Exp. 2015.

47. Faustino-Rocha A, Oliveira PA, Pinho-Oliveira J, Teixeira-Guedes C, Soares-Maia R, da Costa RG, Colaco B, Pires MJ, Colaco J, Ferreira R, Ginja M: Estimation of rat mammary tumor volume using caliper and ultrasonography measurements. Lab Anim (NY). 2013; 42:217-24.

48. Lu R, Wu S, Liu X, Xia Y, Zhang YG, Sun J: Chronic effects of a Salmonella type III secretion effector protein AvrA in vivo. PLoS One. 2010; 5:e10505.

49. Tack DM, Marder EP, Griffin PM, Cieslak PR, Dunn J, Hurd S, Scallan E, Lathrop S, Muse A, Ryan P, et al: Preliminary incidence and trends of infections with pathogens transmitted commonly through food - Foodborne Diseases Active Surveillance Network, 10 U.S. sites, 2015-2018. American Journal of Transplantation. 2019; 19:1859-63.

50. Lu R, Wu S, Zhang YG, Xia Y, Zhou Z, Kato I, Dong H, Bissonnette M, Sun J: Salmonella Protein AvrA Activates the STAT3 Signaling Pathway in Colon Cancer. Neoplasia. 2016; 18:307-16.

51. Kong Y, He M, McAlister T, Seviour R, Forster R: Quantitative fluorescence in situ hybridization of microbial communities in the rumens of cattle fed different diets. Appl Environ Microbiol. 2010; 76:6933-8.

52. Zhang J, Kobert K, Flouri T, Stamatakis A: PEAR: a fast and accurate Illumina Paired-End reAd mergeR. Bioinformatics. 2014; 30:614-20.

53. Glockner FO, Yilmaz P, Quast C, Gerken J, Beccati A, Ciuprina A, Bruns G, Yarza P, Peplies J, Westram $\mathrm{R}$, Ludwig W: 25 years of serving the community with ribosomal RNA gene reference databases and tools. J Biotechnol. 2017; 261:169-76.

54. Edgar RC: Search and clustering orders of magnitude faster than BLAST. Bioinformatics. 2010; 26:2460-1.

55. Xia Y, Sun J, Chen D-G: Statistical analysis of microbiome data with R. Springer; 2018.

56. R Core Team: R: A language and environment for statistical computing. Vienna, Austria: R Foundation for Statistical Computing; 2019.

57. Xia Y, Sun J, Chen D-G: Statistical analysis of microbiome data with R. Springer; 2018.

\section{Figures}


Fig. 1
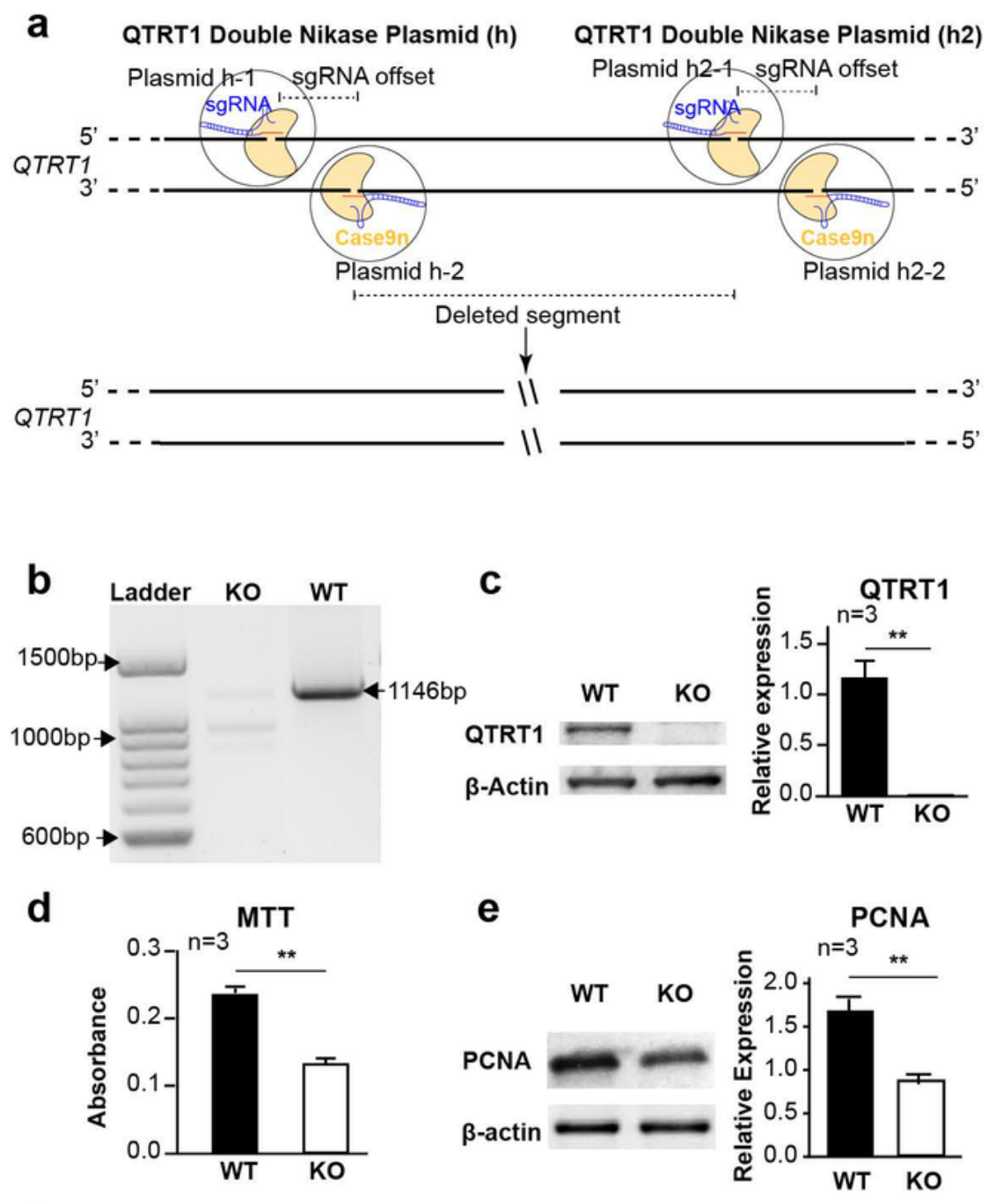

$\mathbf{f}$
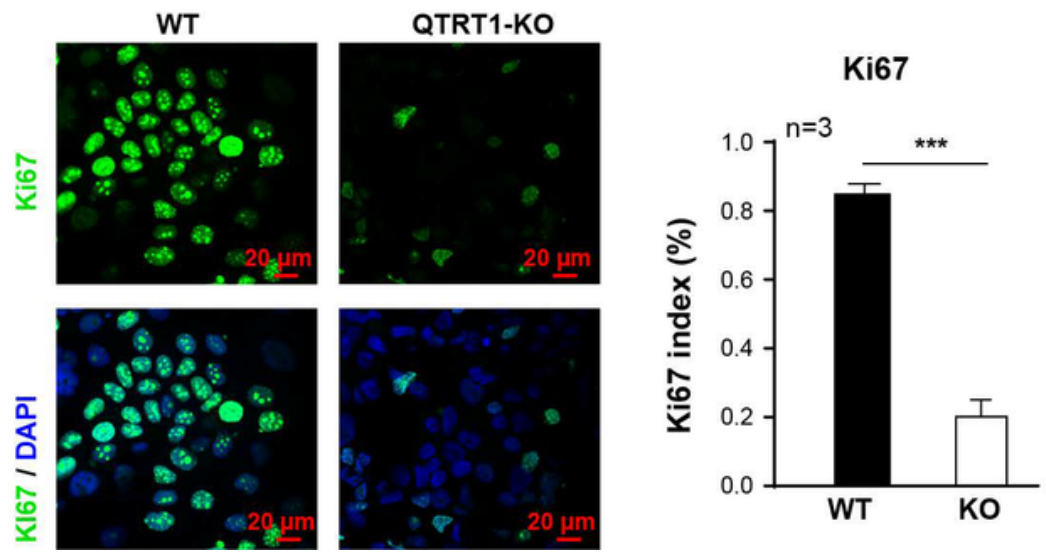

\section{Figure 2}

Knockout of QTRT1 suppressed MCF7 breast cancer cell proliferation. a Schematic illustration of doublestranded DNA breaks using a pair of Cas9 D10A nickases (Cas9n). b The deletion of QTRT1 in MCF7 cells were confirmed using PCR with primers specific for the QTRT1 gene. c Western blot analysis of wildtype (WT), QTRT1-knockout (KO) MCF7 cells generated using Double Nickase Plasmids after treating for 72 hours. Mean \pm SD, $n=3$; ** means P-value $<0.01$, two-tailed Welch's t-test. $d$ MTT assay to show cell 
proliferation of WT and QTRT1-KO MCF7 conducted at $48 \mathrm{~h}$ after seeding the same number of cells. Mean \pm SD, $n=3$; ** means P-value $<0.01$, two-tailed Welch's t-test. e, $f$ Cell proliferation markers of PCNA and Ki67 in WT and QTRT1-KO MCF7 cells were detected using western blot (E) and immunofluorescence staining (F), respectively. Immunofluorescence staining of Ki67 (green) and DAPI (blue) were performed in the cells, and Ki67 index (Ki67 stained cells / total cells) was calculated. Mean \pm SD, $n=3$; ** P-value < 0.01 , *** P-value $<0.001$, two-tailed Welch's t-test.

Fig. 2
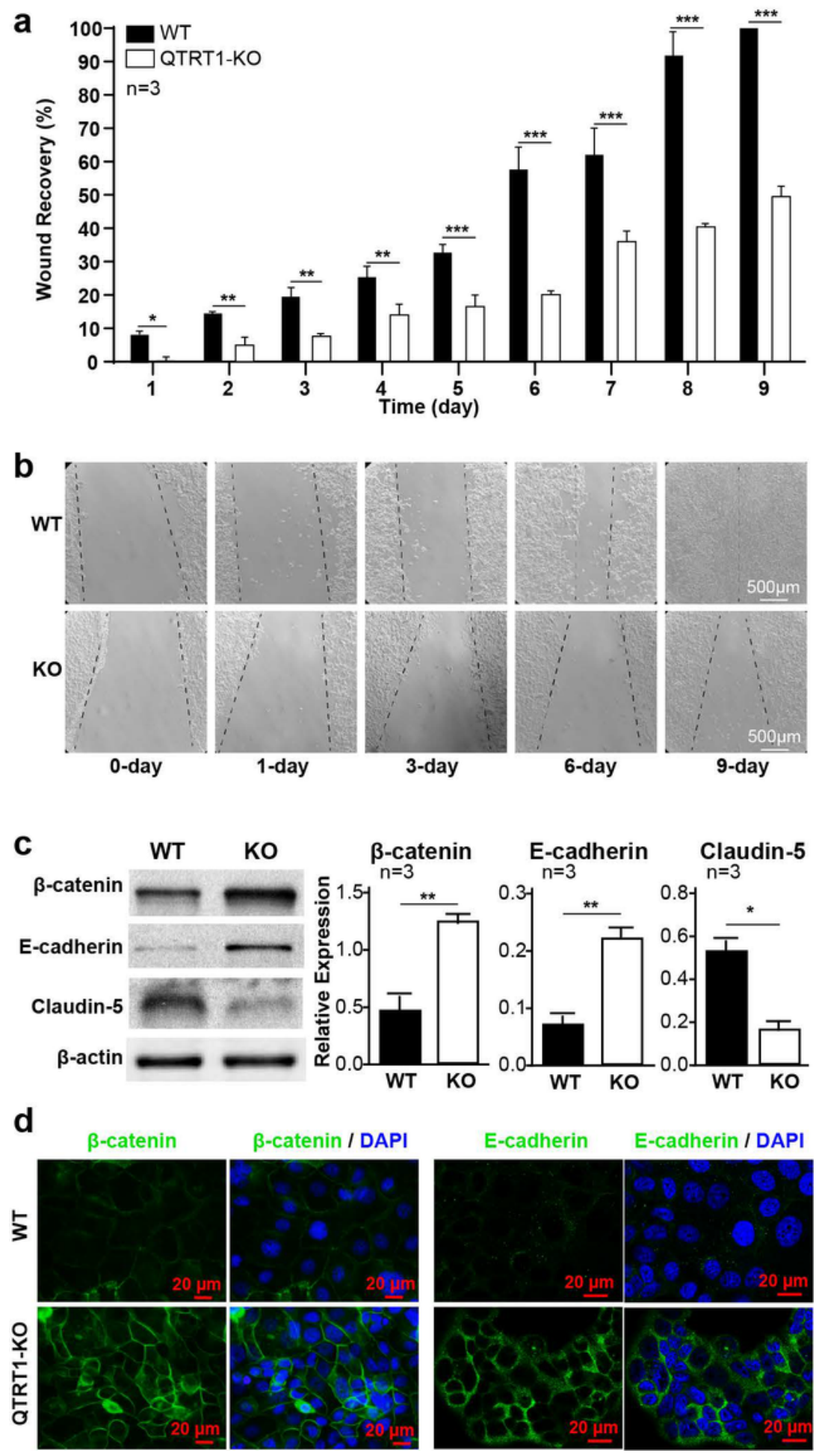

Figure 4 
Knockout of QTRT1 suppressed cell migration and altered cell adhesion and Tight Junctions. a Wound healing analysis of wildtype and QTRT1-KO MCF7 cells was shown as the percentage of scratch closure at day-timepoints. Mean $\pm S D, n=3$; * means P-value $<0.05$, ** means P-value $<0.01$, two-tailed Welch's ttest. b Representative wound healing images were shown. Scale bar is $500 \mu \mathrm{m}$. c Western analysis of tight junction protein E-cadherin, $\beta$-catenin and Caludin- 5 was performed on QTRT1 knockout and WT MCF7 cells. Mean $\pm S D, n=3$; * P-value $<0.05$, ** P-value $<0.01$, two-tailed Welch's t-test. $d$ E-cadherin and $\beta$ catenin immunofluorescence staining showing the protein expression in QTRT1-KO MCF7 cells compared with WT cells. Proteins and DNA were stained with mouse monoclonal anti- $\beta$-catenin/E-cadherin and antimouse Alexa Fluor 488 (green) antibody, and DAPI (blue), respectively. Scale bar is $20 \mu \mathrm{m}$. 
Fig. 3
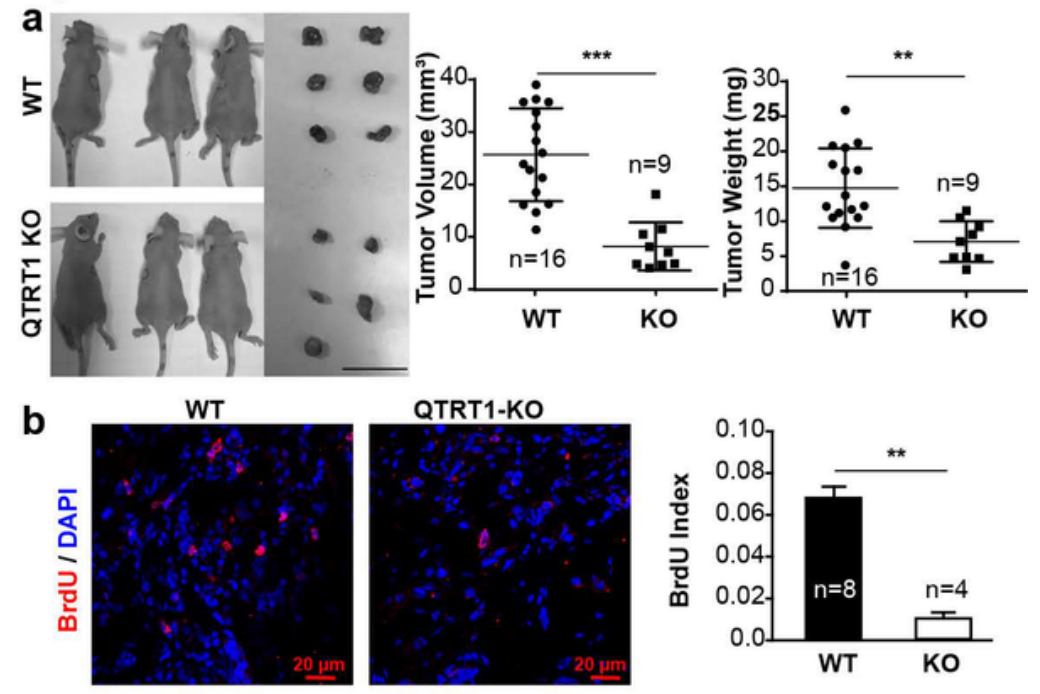

QTRT1-KO

c
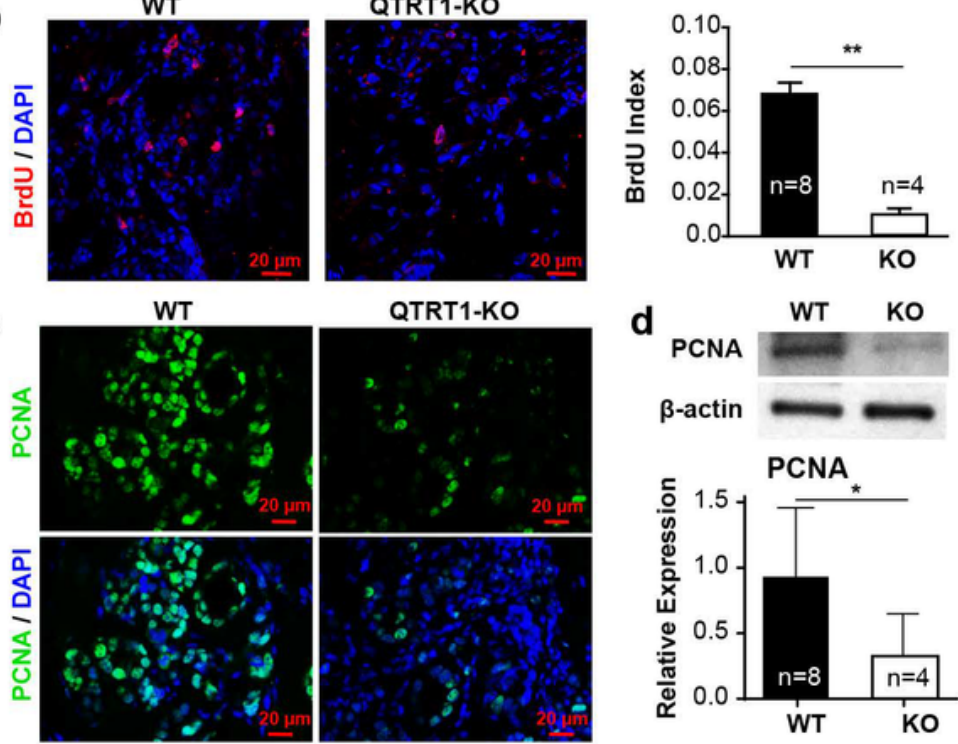

e
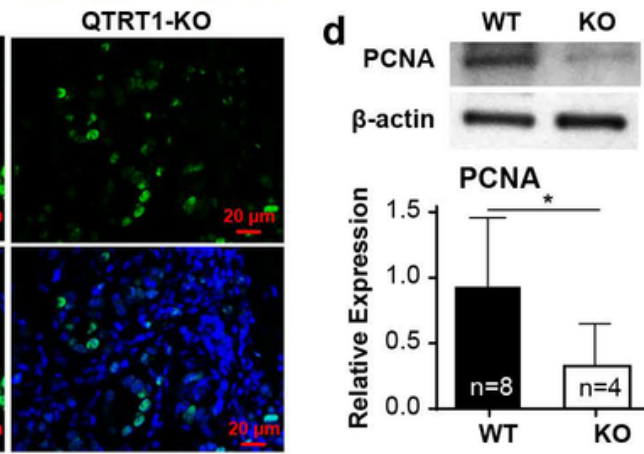

$\beta$-actin
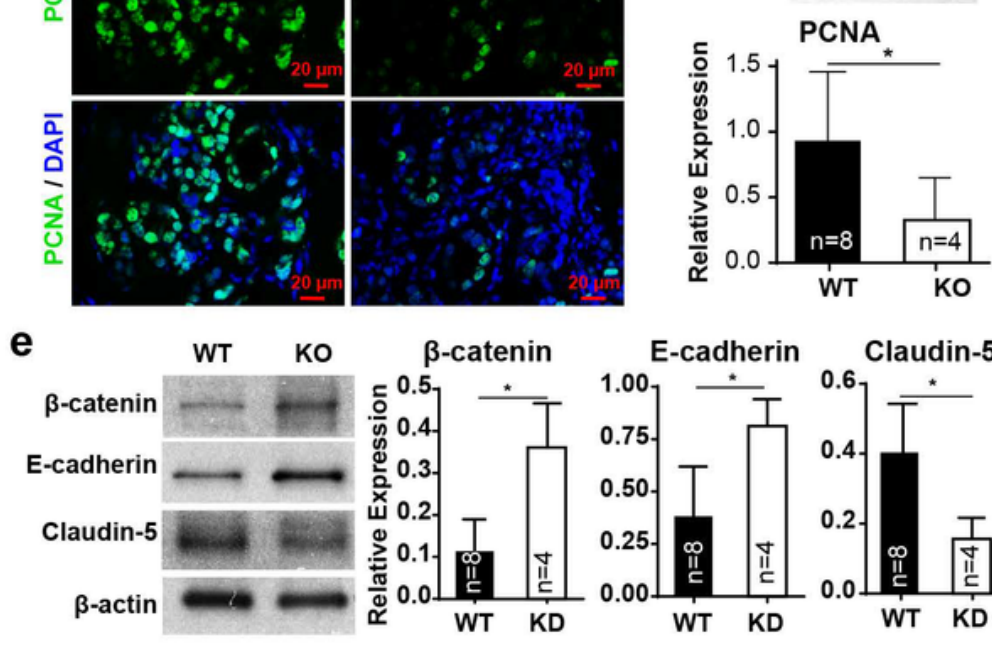

E-cadherin

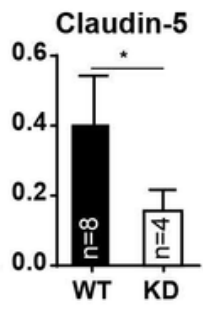

f

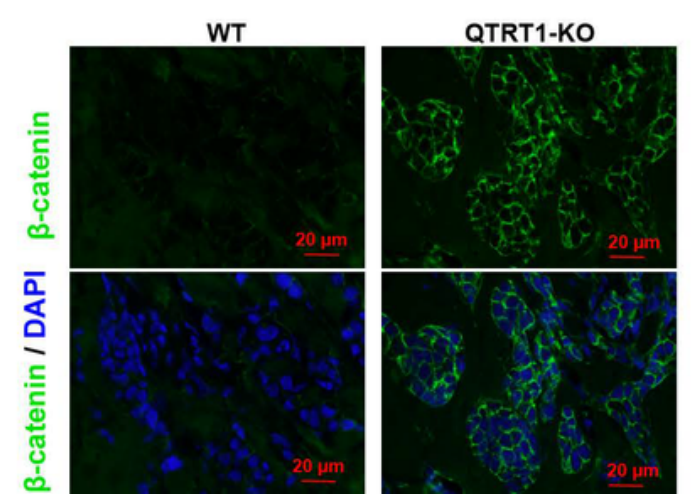

Figure 6

Knockout of QTRT1 suppressed tumor growth and altered Tight Junctions in tumors in vivo. a The WT and QTRT1-KO MCF7 cells were injected into nude mice $(n=10)$, and tumors were harvest 60-day post injection (WT: $n=16 ; K O: n=9$ ). The representative photograph of tumors in vivo and in vitro (left), tumor volume (middle) and tumor weight (right) were shown. The scale bar indicates $10 \mathrm{~mm}$. Each circle represents an individual tumor. Mean $\pm S D, n=16$ or $n=9$; ** P-value $<0.01$, *** P-value $<0.001$, two-tailed 
Welch's t-test. b BrdU immunofluorescence staining and index analysis were performed on the tumors from nude mice injected WT and QTRT1-KO MCF7 cells. BrdU was detected with sheep monoclonal antiBrdU and anti-sheep Alexa Fluor 594 (red) antibody. The scale bar is $20 \mu \mathrm{m}$. Mean $\pm \mathrm{SD}, \mathrm{n}=8$ or $\mathrm{n}=4$; ** $\mathrm{P}$ value $<0.01$, two-tailed Welch's t-test. c Immunocytochemical analysis of PCNA in tumors from nude mice. PCNA and DNA was stained with mouse monoclonal anti-PCNA and anti-mouse Alexa Fluor 488 (green) antibody, and DAPI (blue), respectively. The scale bar is $20 \mu \mathrm{m}$. d Western blot analysis of PCNA in tumors harvest from the mice injected with WT and QTRT1-KO MCF7 cells. The graph shows the band intensities of PCNA proteins. Mean \pm SD $n=8$ or $n=4$; * $P$-value $<0.05$, two-tailed Welch's t-test. e Western blot analysis of tight junction regulators was performed on the tumors isolated from the nude mice model injected with WT and QTRT1-KO MCF7 breast cancer cells. The graphs show the relative expression of the target proteins detected by western blot. Mean $\pm S D, n=8$ or $n=4$; * P-value $<0.05$, two-tailed Welch's $t$ test. $f$ Immunofluorescence staining of $\beta$-catenin was performed on tumors from both QTRT1-KO cells injected mice and WT cells injected mice. Proteins and DNA were stained with mouse monoclonal anti- $\beta$ catenin and anti-mouse Alexa Fluor 488 (green) antibody and DAPI (blue), respectively. Scale bar is $20 \mu \mathrm{m}$. 
Fig. 4

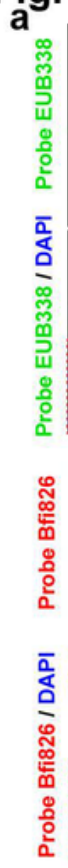

C

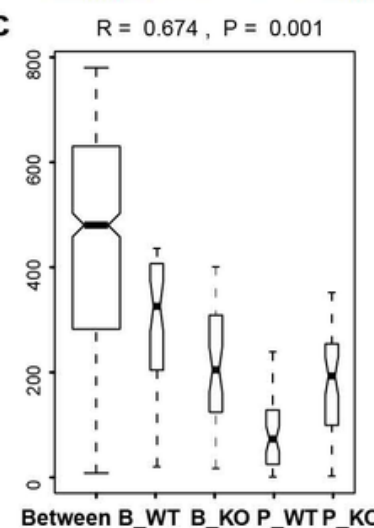

QTRT1-KO
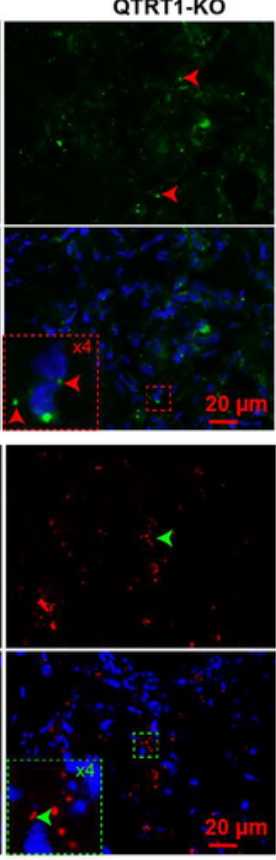

d

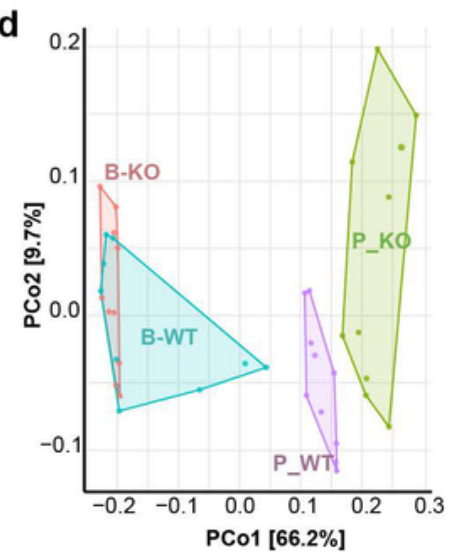

b $\overline{\bar{a}}$
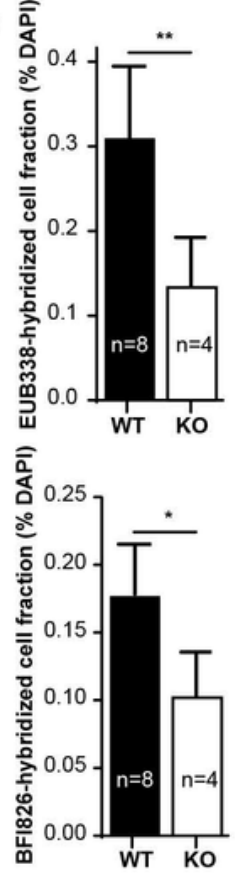

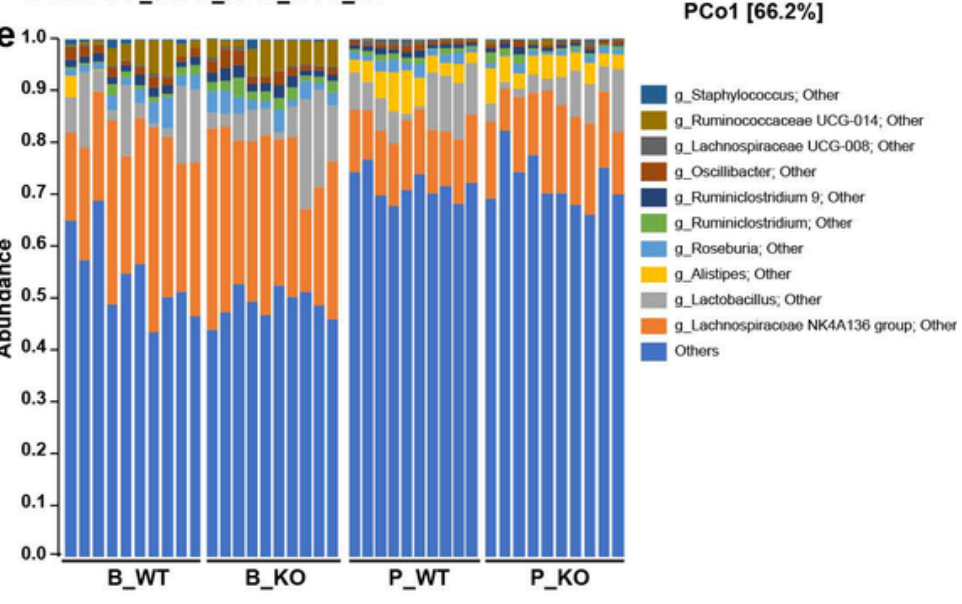

Figure 8

Altered microbiome in tumors and intestine of nude mouse model. a Fluorescence in situ hybridization [4] staining with DAPI (blue), EUB338 (green) and Bfi826 (red) of the tumors from the nude mice injected with wildtype and QTRT1-KO MCF7 breast cancer cells. Scale bar is $20 \mu \mathrm{m}$. b Relative bacteria staining was calculated as probe-hybridized cell / DAPI-stained cells. Mean \pm SD $n=7$ or $n=4$; * means P-value < 0.05 , ** means P-value < 0.01, two-tailed Welch's t-test. c Plots of between and within means of Bray- 
Curtis Dissimilarity. The analysis of similarity (ANOSIM) was performed on between (Between) and within groups of before QTRT1-KO cell injection (B_KO), before WT cell injection (B_WT), post QTRT1-KO cell injection (P_KO) and post WT cell injection (P_WT) based on the Bray-Curtis Dissimilarity analysis. $n=10$ per group. $d$ The principal coordinates analysis (PCOA) plot of the mouse feces was produced to inspect the homogeneity of multivariate dispersions. The samples collected before (B_WT and B_KO) and post (P_WT and P_KO) cell injection were colored in the illustration. $n=10$ per group. e Relative bacterial abundance in species level ( $s_{-}$; the unidentified species were named with super level and other, genus/g, family/f_) of feces collected from nude mice before (B_WT and B_KO) and post (P_WT and P_KO) injection of wildtype (WT) and QTRT1-KO MCF7 cells was shown with the top 30 species, and lower ones were grouped as "Others". Species was colored using the key as list in the right side. Each bar represents individual mouse ( $\mathrm{n}=10$ each group). 
Fig. 5
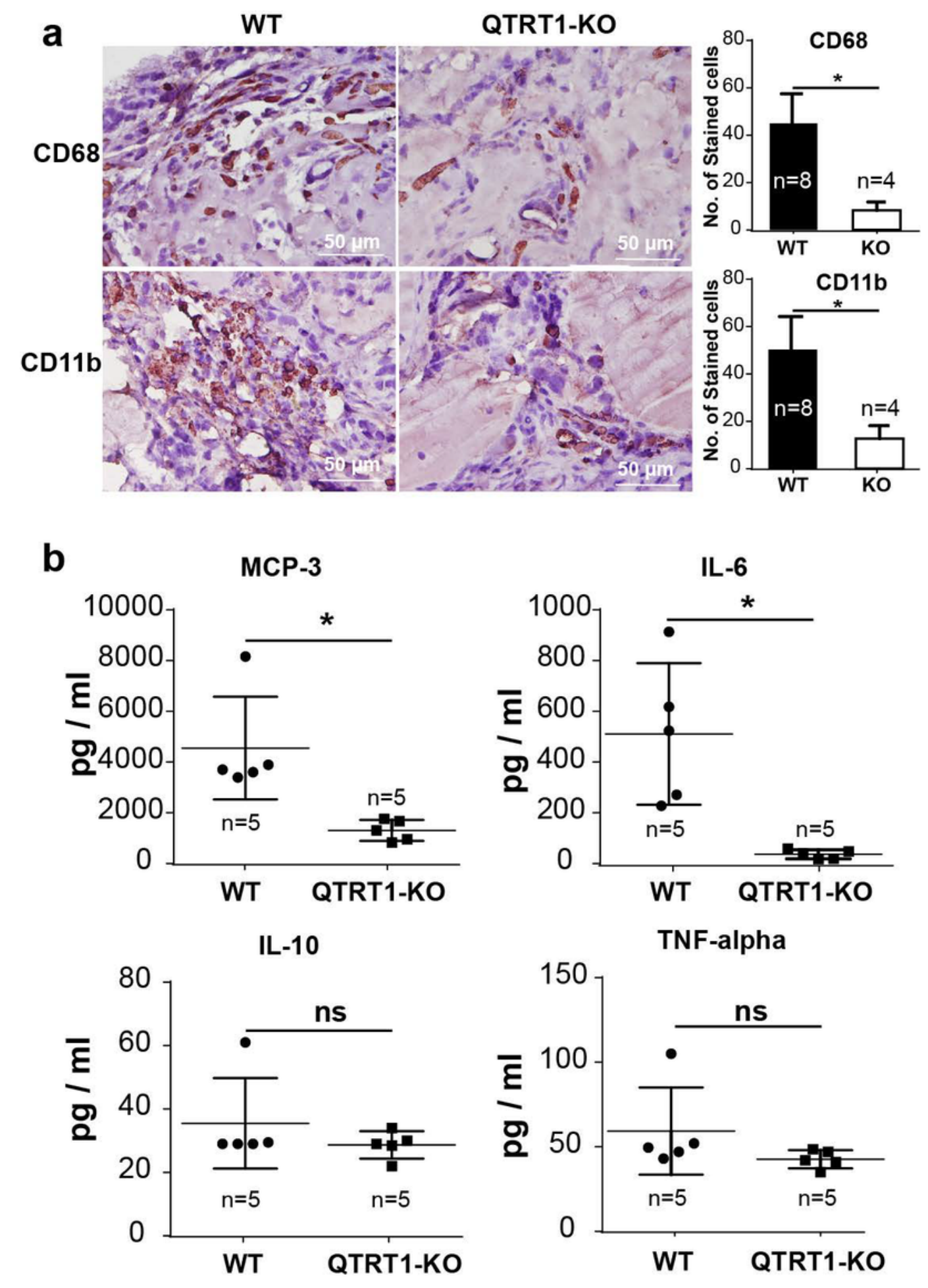

Figure 10

Inflammation regulators in tumors and plasma of the nude mice. a Immunostaining of inflammationrelated cells in tumors from nude mouse with anti-CD68 and anti-CD11b. The number of stained cells were calculated in each visual filed. and Scale bar is $50 \mu$ m. Mean $\pm S D, n=8$ or $n=4$; * means P-value < 0.05 , two-tailed Welch's t-test. b Mouse cytokine level in plasma of nude mice injected WT and QTRT1-KO 
MCF7 breast cancer cells was determined by multiplex bead-based assays for 26 cytokines and chemokines. Mean $\pm S D, n=5$; * P-value $<0.05$, ns means no significance.

Fig. 6
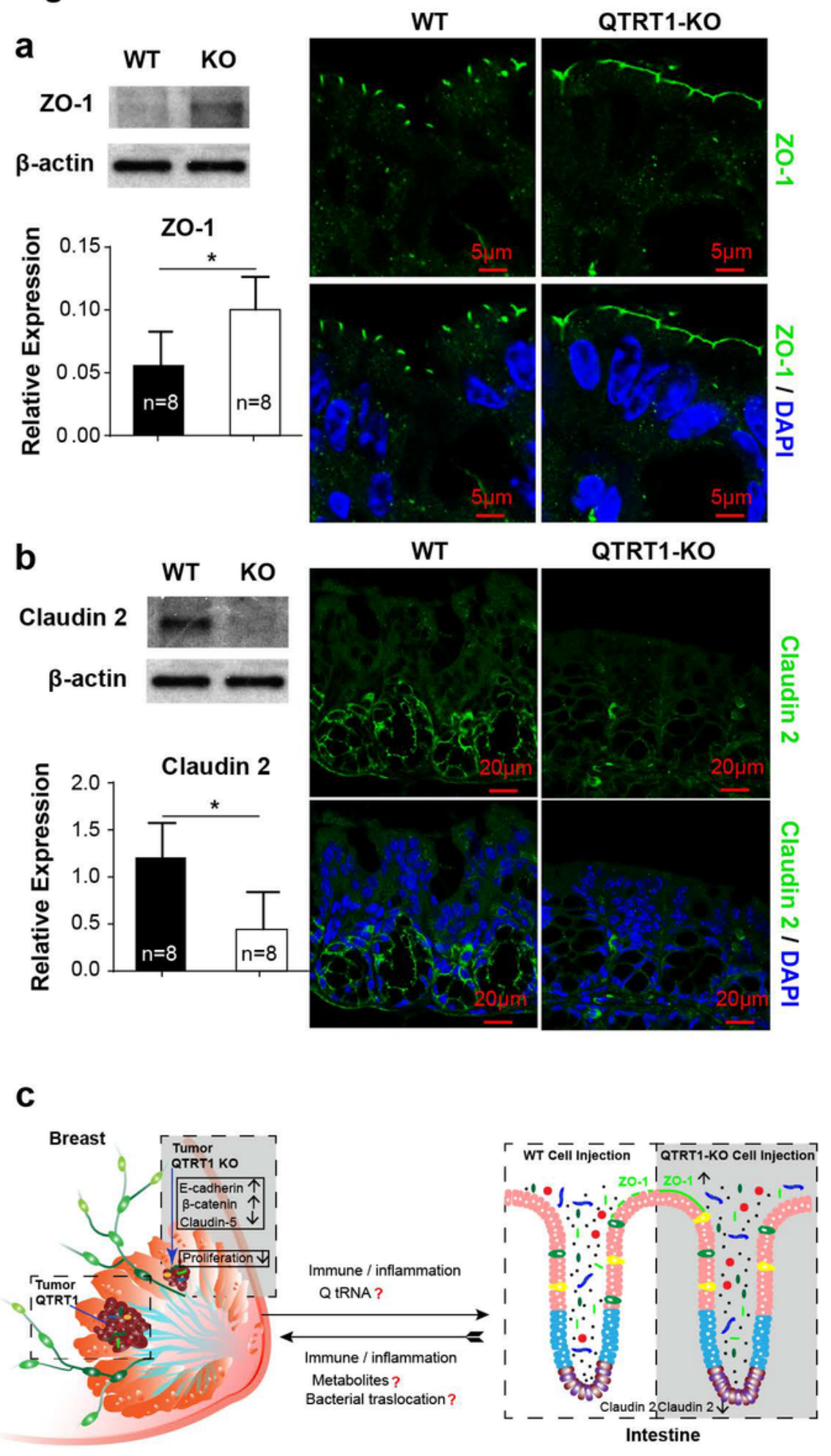

Figure 11

Altered tight junction proteins expression in colon of nude mouse model. Western blot analysis and immunofluorescence staining of ZO-1 (a) and Claudin 2 (b) were performed on the colons from the nude mice injected with WT and QTRT1-KO MCF7 breast cancer cells. Proteins and DNA were stained with 
rabbit monoclonal anti-ZO-1/ mouse monoclonal anti-Claudin 2 and anti-mouse Alexa Fluor 488 (green) antibody, and DAPI (blue), respectively. Scale bar is $5 \mu \mathrm{m}$ in (a) and $20 \mu \mathrm{m}$ in (b). The graphs show the relative expression of the target proteins detected by western blot. Mean $\pm S D, n=8$; * P-value $<0.05$, twotailed Welch's t-test. c The working model of QTRT1 knockout in breast cancer and intestine. Knockout of QTRT1 inhibited the beast tumor proliferation and growth by altering the expression of Claudin-5, Ecadherin and $\beta$-catenin in breast, and changed microenvironment of tumors. Meanwhile, the altered expression of tight junction proteins Claudin 2 and ZO-1, and altered bacterial community was found in the intestine from the mouse model. It is unknown what was the role of Q TRNA and metabolites in the process, and whether the bacterial translocation from the intestine to the breast was happened.

\section{Supplementary Files}

This is a list of supplementary files associated with this preprint. Click to download.

- SFiguresandlegends.pdf

- SFiguresandlegends.pdf 